\title{
Immunohistochemical Study of Mitochondrial Ferritin in the Midbrain of Patients with Progressive Supranuclear Palsy
}

\author{
Zulzikry Hafiz Abu Bakar', Tomoko Kato ${ }^{1}$, Daijiro Yanagisawa ${ }^{1}$, Jean-Pierre Bellier ${ }^{1}$, \\ Ken-ichi Mukaisho ${ }^{2,3}$ and Ikuo Tooyama ${ }^{1}$ \\ ${ }^{1}$ Molecular Neuroscience Research Center, Shiga University of Medical Science, ${ }^{2}$ Department of Pathology, Shiga University \\ of Medical Science and ${ }^{3}$ Medical and Nursing Education Center, Shiga University of Medical Science, Seta Tsukinowa-cho, \\ Otsu 520-2192, Japan
}

Received February 8, 2021; accepted May 26, 2021; published online June 23, 2021

\begin{abstract}
Mitochondrial ferritin (FtMt) is a novel ferritin that is localized in the mitochondria. FtMt expression is low in the liver and spleen, and high in the heart, testis, and brain. We previously detected FtMt in dopaminergic neurons in the substantia nigra pars compacta (SNc) in human and monkey midbrains. We investigated the localization and expression of FtMt in the midbrain of patients with progressive supranuclear palsy (PSP) and controls using a monoclonal antibody (C65-2) against human FtMt. FtMt immunoreactivity was weakly detected in neuromelanin-containing neurons in the SNc and ventral tegmental area (VTA) of control cases compared with PSP, which exhibited a remarkable increase in FtMt immunoreactivity. Preincubation of $\mathrm{C} 65-2$ with the immunizing FtMt peptide significantly reduced the staining, indicating the specificity of $665-2$. Several puncta were observed outside the neurons of PSP, in contrast with the control cases. Double immunofluorescence histochemistry for FtMt and tyrosine hydroxylase (TH), glial fibrillary acidic protein, and Iba1 showed localization of FtMt in dopaminergic neurons, microglia, and astrocytes in PSP. Furthermore, FtMt immunoreactivity was detected in a few TH-negative neurons. In the SNc and VTA, FtMt immunoreactivity colocalized with phosphorylated tau immunoreactivity. Our results indicate that FtMt is involved in the pathology of PSP. Clarifying the involvement of FtMt in PSP is of great interest.
\end{abstract}

Key words: dopaminergic neuron, immunohistochemistry, mitochondrial ferritin, progressive supranuclear palsy

\section{Introduction}

Progressive supranuclear palsy (PSP) is a neurodegenerative disorder that primarily affects motor function, causing eye movement disturbance, parkinsonism, and subcortical dementia $[4,6]$. As it progresses, blurry vision and difficulty controlling eye movement are characteristics of PSP [4-6]. Annually, the number of cases of PSP is 5-6

Correspondence to: Ikuo Tooyama, MD, PhD, Professor, Molecular Neuroscience Research Center, Shiga University of Medical Science, Seta Tsukinowa-cho, Otsu 520-2192, Japan.

E-mail: kinchan@belle.shiga-med.ac.jp per 100,000 individuals $[15,18]$. Neuropathologically, PSP shows loss of dopaminergic neurons in the substantia nigra pars compacta $(\mathrm{SNc})$ that project to the putamen [8]. In addition, aberrant tau has been recognized as a pathological feature of PSP, with aggregates of the 4-repeat isoform forming in neurons, astrocytes, and oligodendrocytes in the basal ganglia, including the substantia nigra $[4,6,8,11]$. However, the mechanisms responsible for the emergence of tau aggregates remain unclear.

Excessive iron is considered to increase the propensity of tau to aggregate [20]. In particular, oxidized iron $\left(\mathrm{Fe}^{3+}\right)$ was found to be involved in promoting tau aggregation, which was reversed when $\mathrm{Fe}^{3+}$ levels were reduced 
Table 1. Clinicopathological data of the patients in this study

\begin{tabular}{|c|c|c|c|c|c|}
\hline Cases & Age (years) & Sex & Postmortem delay (hr) & Clinical diagnosis & FtMt-staining** \\
\hline Control-1 & 52 & M & 10.0 & Malignant lymphoma & + \\
\hline Control-2 & 57 & $\mathrm{~F}$ & 49.0 & Cancer of unknown origin & $+/-$ \\
\hline Control-3 & 66 & M & 20.0 & Prostate cancer & $+/-$ \\
\hline Control-4 & 64 & M & 12.0 & Prostate cancer/subdural hemorrhage & + \\
\hline PSP-1 & 47 & M & $<12.0^{*}$ & Progressive supranuclear palsy & ++ \\
\hline PSP-2 & 69 & M & 13.0 & Progressive supranuclear palsy & +++ \\
\hline PSP-3 & 68 & $\mathrm{~F}$ & 5.0 & Progressive supranuclear palsy & ++ \\
\hline PSP-4 & 76 & M & 10.0 & Progressive supranuclear palsy & +++ \\
\hline
\end{tabular}

* Accurate postmortem delay was not assessed.

** Staining intensity of FtMt expression in the substantia nigra: $+/-$, very weak; + , weak; ++ , strong; +++ , very strong.

FtMt: mitochondrial ferritin; PSP: progressive supranuclear palsy.

[24]. The formation of $\mathrm{Fe}^{3+}$ through the Fenton reaction involves the induction of reactive oxygen species (ROS) formation [7], and oxidative stress may cause PSP [3]. Therefore, investigating iron metabolism in the basal ganglia of patients with PSP is important.

Mitochondrial ferritin (FtMt) is a novel protein localized in the mitochondria [16]. It has $\sim 79 \%$ homology with H-chain ferritin (FTH), which is ubiquitously present in the cytoplasm [16]. Although both proteins acquire ferroxidase activity and possess similar iron storage properties, FtMt expression seems to correlate relatively well with mitochondrial abundance and not with iron metabolism [9, 17]. FtMt is highly expressed in spermatids and interstitial cells, neuronal cells, and other cells, with the exception of hepatocytes, splenocytes, and myocytes, which contain abundant iron $[9,16,17]$. We previously reported the expression of FtMt in dopaminergic neurons in the $\mathrm{SNc}$ of the human and monkey midbrain using a novel mouse monoclonal antibody clone, C65-2 [28]. Previous studies have reported that FtMt expression increases in neurodegenerative diseases, such as Alzheimer's and Parkinson's disease $[12,25]$. However, its involvement in the pathogenesis of neurodegenerative diseases remains unclear.

In this study, we investigated the localization and expression of FtMt in the midbrain of patients with PSP and control cases by immunohistochemistry using a monoclonal antibody (C65-2) against human FtMt.

\section{Materials and Methods}

\section{Human brain sample}

This study complied with the Declaration of Helsinki and was approved by the Ethics Committee of Shiga University of Medical Science (reference number R2016-026). Postmortem midbrain tissues from normal individuals $(\mathrm{n}=$ 4) and patients with PSP $(n=4)$ were obtained from the brain bank at Shiga University of Medical Science. The tissues were collected with the patients' consent. Postmortem midbrains were fixed in formalin and embedded in paraffin. Paraffin blocks were cut to $5 \mu \mathrm{m}$. Tissue preparation was performed as described in a previous report [14]. The clinicopathological features of the patients are presented in Table 1.

\section{Immunohistochemistry}

Immunohistochemical analyses were conducted as previously described $[1,27,28]$, with some modifications. Deparaffinized sections were washed several times before being incubated in a $1 \%$ hydrogen peroxide solution for 20 min. Heat-induced epitope retrieval was performed using a microwave with $1 \mathrm{mM}$ ethylenediaminetetraacetic acid (EDTA) for $4 \mathrm{~min}$. Tissue sections were blocked with $2 \%$ bovine serum albumin (BSA) in $0.1 \mathrm{M}$ phosphate-buffered saline containing $0.3 \%$ Triton X-100 (pH 7.4; PBST) for 30 min after several washes with PBST. The sections were incubated with the mouse monoclonal anti-FtMt antibody (clone $\mathrm{C} 65-2 ; 2 \mu \mathrm{g} / \mathrm{mL}$ ) [28] overnight at $4^{\circ} \mathrm{C}$, followed by biotinylated anti-mouse IgM (1: 500) for $1 \mathrm{hr}$. After washing with PBST, the sections were incubated with the avidin-biotin-peroxidase complex (Vectastain ABC Elite kit; 1:3000; Vector Laboratories, Burlingame, CA, USA) for $1 \mathrm{hr}$ at room temperature. After several washes, peroxidase-bound sections were developed in $0.02 \% 3,3$ diamine-benzidine tetrahydrochloride (DAB) containing $0.3 \%$ nickel ammonium sulfate in $50 \mathrm{mM}$ Tris- $\mathrm{HCl}(\mathrm{pH}$ 7.6) with $0.005 \%$ hydrogen peroxide for $10 \mathrm{~min}$. Some sections were counterstained with neutral red stain.

Digital images of the sections were acquired with a Nikon-D90 digital camera (Tokyo, Japan) on an Olympus Microscope BX50 (Tokyo, Japan).

\section{Double-immunofluorescence histochemistry}

Deparaffinized sections were washed several times prior to heat-induced epitope retrieval using a microwave with $1 \mathrm{mM}$ EDTA for $4 \mathrm{~min}$. After several washes with PBST sections were blocked with $2 \%$ BSA in $0.1 \mathrm{M}$ PBST for $30 \mathrm{~min}$. The sections were incubated overnight with mouse monoclonal anti-FtMt antibody $(2 \mu \mathrm{g} / \mathrm{ml})$ and rabbit polyclonal antibody against TH (1:1000; Millipore, Temecula, CA, USA), GFAP (1:1000; Abcam, Cambridge, 


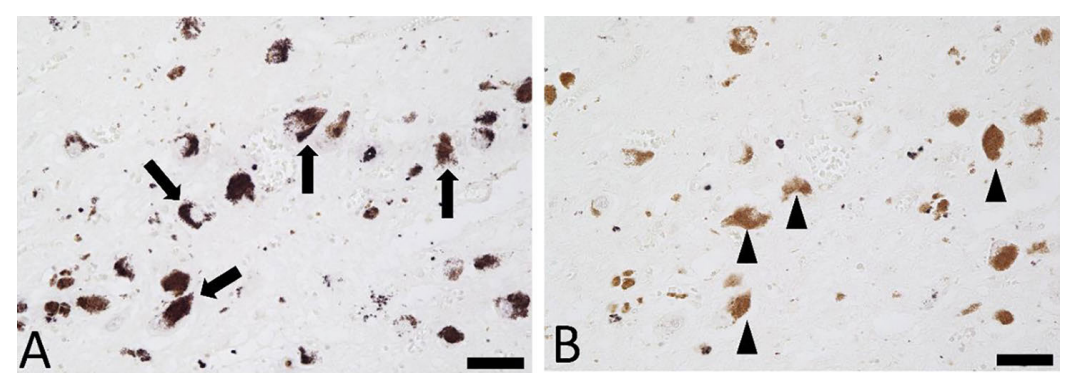

Fig. 1. Absorption test for C65-2 antibody in human brain sections (PSP-4). A: Immunostaining for mitochondrial ferritin (FtMt) in the substantia nigra pars compacta ( $\mathrm{SNc}$ ) of a patient with progressive supranuclear palsy (PSP). FtMt is stained dark purple (arrows). B: The immunostaining was notably reduced when the antibody was pre-absorbed with human FtMt peptide. Brown granules indicating neuromelanin are observed (arrowheads). Bars $=$ $50 \mu \mathrm{m}$.

UK), or Iba1 (1:500; Wako Pure Chemical Industries, Osaka, Japan) at $4^{\circ} \mathrm{C}$. In addition, some sections were incubated overnight with mouse monoclonal anti-FtMt antibody $(2 \mu \mathrm{g} / \mathrm{ml})$ and rat monoclonal anti-phosphorylated Tau S199 (pTau; 1:200; Wako Pure Chemical Industries) at $4^{\circ} \mathrm{C}$. After washing with $10 \mathrm{mM}$ PBS, sections were then incubated for $1 \mathrm{hr}$ with Alexa Fluor 555-labeled donkey anti-mouse IgG (1:500, Invitrogen, Frederick, MD, USA) for FtMt and Alexa Fluor 647-labelled goat anti-rabbit IgG (1:500, Invitrogen) for TH, Alexa Fluor 488-labelled goat anti-rabbit IgG (1:500, Invitrogen) for GFAP and Iba1 or Alexa Fluor 488-labelled goat anti-rat (1:200, Invitrogen) for pTau. Following incubation, the sections were washed several times with $10 \mathrm{mM}$ PBS. Sections were then incubated for $50 \mathrm{~s}$ with true black solution (1:40 diluted with $70 \%$ ethanol; Biotium, Fremont, CA, USA) to reduce background fluorescence. After washing with 0.1 M PBS and distilled water, the sections were cover-slipped with Immunomount (Thermo Fisher Science, MI, USA).

Digital images of the sections were acquired with a Leica TCS SP8 confocal laser scanning microscope (Wetzlar, Germany) on a Leica DMi8 microscope.

\section{Absorption test}

The absorption test was conducted as previously described [1,28], with some modifications. Deparaffinized sections were washed several times before being incubated in a $1 \%$ hydrogen peroxide solution for $20 \mathrm{~min}$. Heatinduced epitope retrieval was performed using microwave irradiation with $1 \mathrm{mM}$ EDTA for $4 \mathrm{~min}$. After several washes with PBST, the sections were blocked with $2 \%$ BSA in PBST for $30 \mathrm{~min}$. The sections were incubated with pre-absorbed C65-2 antibody with FtMt peptide (97.8 $\mu \mathrm{g} / \mathrm{mL} ; 1: 8$, C65-2, FtMt peptide) overnight at $4^{\circ} \mathrm{C}$. After washing with PBST, the sections were immunostained using previously described methods.

Data are presented as mean \pm standard error of mean (SEM). The statistical significance between two groups was analyzed by an unpaired t-test using GraphPad Prism 7 (GraphPad Software, La Jolla, CA, USA).

\section{Results}

\section{Characterization of C65-2 as the human FtMt monoclonal antibody in human brain sections}

The characterization of the human FtMt monoclonal antibody $\mathrm{C} 65-2$ was described in a previous study from our group [28]. Briefly, the C65-2 antibody detected a single band of approximately $22 \mathrm{kDa}$, corresponding to the molecular weight of FtMt in total crude protein [28]. No bands were observed when the antibody was pre-absorbed with the FtMt peptide or when no primary antibody was used [28]. Although FtMt has high homology with H-type ferritin (FTH), a western blot analysis revealed that C65-2 did not cross-react with FTH [28].

In this study, we performed an immune-absorption test. The results of this test are shown in Figure 1. FtMt immunoreactivity was mainly detected in neuromelanincontaining neurons in the SNc (Fig. 1A). The positive signals were dark purple with nickel enhancement (arrows in Fig. 1A). When sections were stained using the C65-2 antibody pre-absorbed with the immunizing FtMt peptide, the staining was significantly reduced (Fig. 1B). Only brown granules indicating neuromelanin were observed (arrowheads in Fig. 1B). Thus, human FtMt was specifically detected using C65-2 antibody.

\section{Distribution of FtMt in the midbrains of patients with PSP and controls}

In control cases, FtMt immunoreactivity was weakly detected in neurons in the SNc (Fig. 2A and B) as well as in the ventral tegmental area (VTA) (Fig. 2C and D). FtMt immunoreactivity was localized to the cell bodies in neuromelanin-positive neurons in the $\mathrm{SNc}$ (arrows in Fig. 2B) and VTA (arrows in Fig. 2D). A few FtMtnegative and neuromelanin-positive neurons were intermingled (arrowhead in Fig. 2D).

In PSP cases, FtMt immunoreactivity was dramatically increased in the SNc (Fig. 2E and F) and VTA (Fig. $2 \mathrm{G}$ and $\mathrm{H}$ ). Most FtMt-immunoreactivities were detected in neuromelanin-positive cells (large arrows in Fig. 2F and 


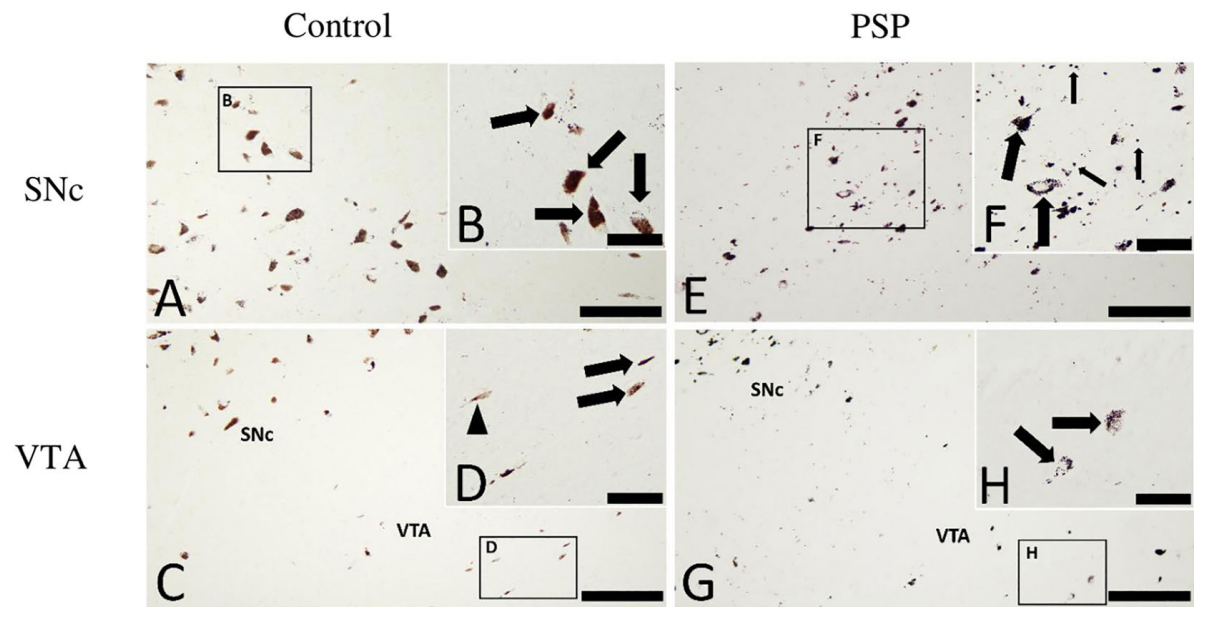

Fig. 2. Immunohistochemical analysis for human mitochondrial ferritin (FtMt) in the substantia nigra pars compacta (SNc) and ventral tegmental area (VTA) of a control case (Control-1; A-D) and a patient with PSP (PSP-2; E-H). A: Distribution of FtMt immunoreactivity in the SNc of a control case. FtMt-immunoreactivity is localized in neuromelanin-positive neurons. B: High-magnification image of the boxed area B in A. FtMt-immunoreactivity was present in cell bodies of neuromelanin-positive neurons (large arrows). C: Distribution of FtMt immunoreactivity in the VTA of a control case. D: High-magnification image of the boxed area $\mathbf{D}$ in C. FtMt-immunoreactivity was weakly observed in neuromelanin-positive cells (arrows). Some FtMt-negative neurons are also visible (arrowhead). E: Distribution of FtMt-immunoreactivity in the SNc of a patient with PSP. The staining intensity of FtMt is increased. F: High-magnification image of the boxed area $\mathbf{F}$ in $\mathbf{E}$. FtMt-immunoreactivity in cell bodies of neuromelanin-positive cells (large arrows) and small dots outside neuromelanin-positive neurons (small arrows). G: FtMt-immunoreactivity in the VTA of a patient with PSP. H: High-magnification image of the boxed area $\mathbf{H}$ in $\mathbf{G}$. FtMt-immunoreactivity is detected in neuromelanin-positive cells (arrows) in the VTA. Bars $=200$ $\mu \mathrm{m}(\mathbf{A}, \mathbf{C}, \mathbf{E}, \mathbf{G})$ and $50 \mu \mathrm{m}(\mathbf{B}, \mathbf{D}, \mathbf{F}, \mathbf{H})$.

H). In addition, many immunoreactive dots were observed outside the neurons, particularly in the SNc (small arrows in Fig. 2F).

\section{Localization of FtMt in dopaminergic neurons}

We performed double immunofluorescence histochemistry of FtMt and TH in the midbrain sections of control cases and patients with PSP (Fig. 3). In control cases, FtMt immunoreactivity was weakly expressed within the SNc (Fig. 3A) and VTA. Almost all FtMt-immunoreactive dots were localized in TH-immunoreactive neurons (arrows in Fig. 3A, B, and C). In PSP cases, the intensity of FtMt immunoreactivity increased in the SNc (Fig. 3D) and VTA. FtMt-immunoreactive dots were localized in THimmunoreactive neurons (arrows in Fig. 3D, E, and F). In addition, FtMt-immunoreactive dots were observed around the TH-immunoreactive neurons (Fig. 3D, E and F). The small dots did not colocalize with TH (Fig. 3D, E and F).

The densities of TH-positive neurons in control cases and PSP patients were 153.35 per $\mathrm{mm}^{2}$ and $50.3 \mathrm{per} / \mathrm{mm}^{2}$, respectively ( $p=0.07$, Fig. $3 \mathrm{G}$ ). And the densities of FtMtpositive neurons in control cases and PSP patients were 124.3 per $\mathrm{mm}^{2}$ and $62.0 \mathrm{per} / \mathrm{mm}^{2}$, respectively $(p=0.29$, Fig. $3 \mathrm{H})$. Although the densities of TH-positive neurons and FtMt-positive neurons have a tendency to be reduced in PSP cases, no significant difference was seen.

Concerning the localization of FtMt and TH, 86.2\% of TH-immunoreactive neurons were colocalized with FtMt in control cases, and $77.5 \%$ of TH-immunoreactive neurons were colocalized with FtMt (Fig. 3I). The ratio of double- positive neurons for FtMt and $\mathrm{TH}$ to total TH-positive neurons was not significantly different between the control and PSP cases $(p=0.343)$. The ratio of FtMt-positive and TH-negative neurons to total FtMt-positive neurons was $13.8 \%$ in control cases, and $38.9 \%$ of FtMt-positive neurons were TH-negative in PSP cases (Fig. 3J). The ratio of FtMt-positive and TH-negative neurons to total FtMt-positive neurons was significantly increased in PSP cases $(p=0.018)$.

\section{Localization of FtMt in astrocytes and microglia}

We used double immunofluorescence histochemistry of FtMt and glial markers (GFAP for astrocytes and Iba1 for microglia) in the midbrain of patients with PSP. Some small FtMt-immunoreactive dots were observed in GFAPimmunoreactive astrocytes (arrow in Fig. 4A, B, C) and Iba1-immunoreactive microglia (arrow in Fig. 4D, E, F).

\section{Co-localization of FtMt and phosphorylated tau}

Double immunofluorescence histochemistry of FtMt and pTau was performed in the midbrain of patients with PSP (Fig. 5). There was no FtMt-immunoreactivity within the superior colliculus (arrowheads in Fig. 5A and A'). However, many pTau-immunoreactive structures were observed in the superior colliculus (arrowheads in Fig. 5B and B'). Subsequently, there was no colocalization of both proteins in the superior colliculus (arrowheads in Fig. 5C and $C^{\prime}$ ).

In the SNc (Fig. 5D) and VTA (Fig. 5G), many FtMtimmunoreactive structures were observed. Many pTau- 

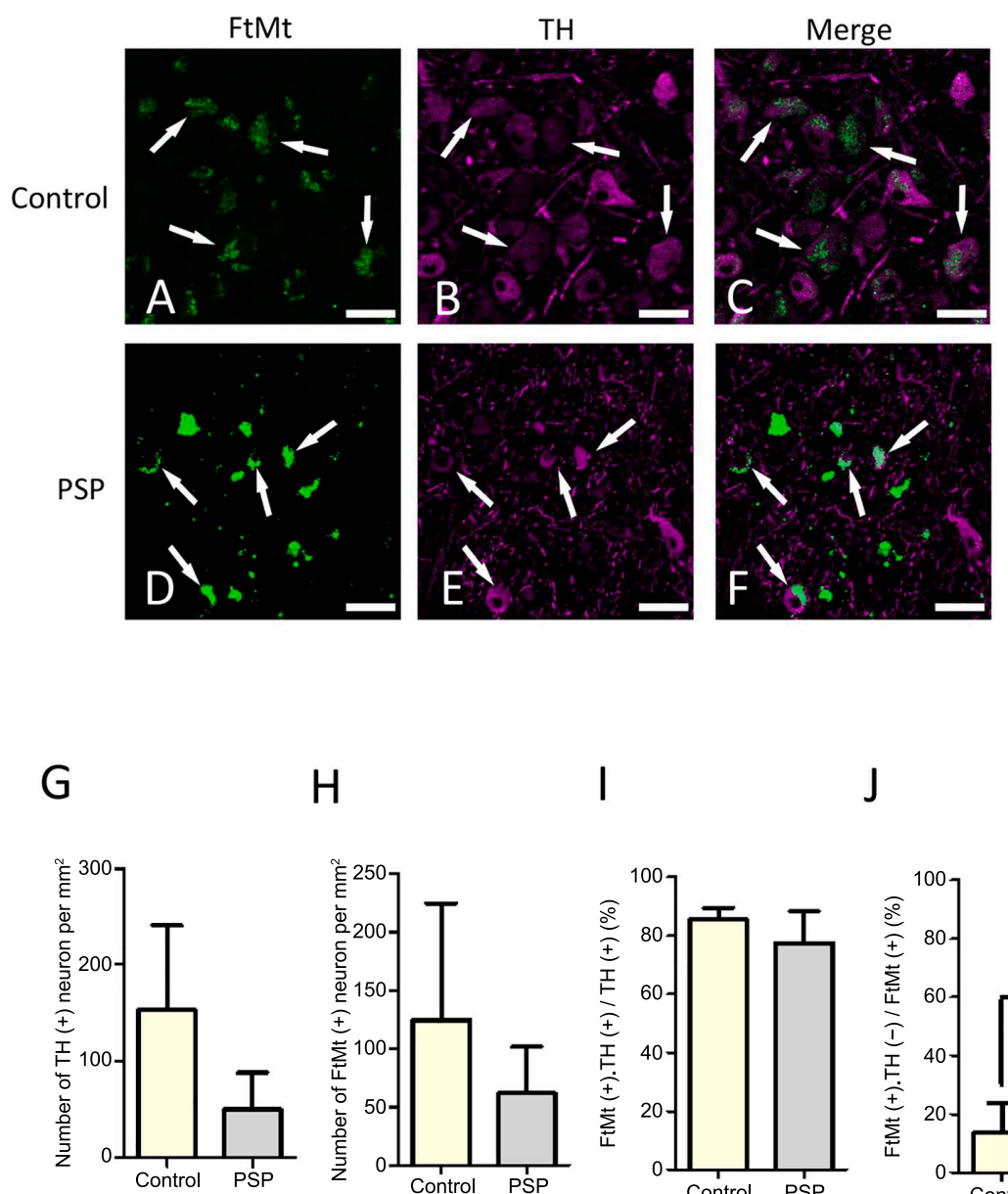

$\mathrm{H}$

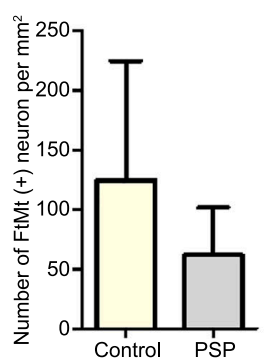

I

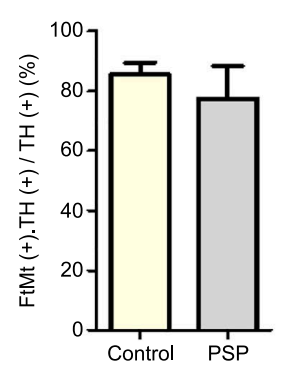

J

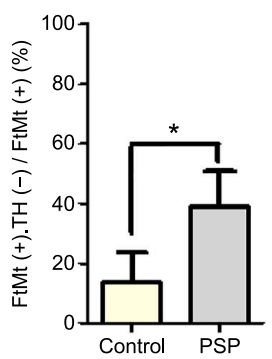

Fig. 3. Double immunofluorescence histochemistry for human mitochondrial ferritin (FtMt) and tyrosine hydroxylase (TH) in the substantia nigra pars compacta $(\mathrm{SNc})$ of the control case (Control-3; A-C) and a patient with PSP (PSP-2; D-F). A: FtMt-immunoreactivity in the SNc of a control case. FtMt-immunoreactive dots are observed (arrow). B: TH-immunoreactive neurons in the SNc (arrow). C: Merged image of FtMt and TH-immunoreactivity in the SNc. FtMt-immunoreactive dots are localized in TH-positive neurons (arrow). D: FtMt-immunoreactivity in the SNc of a PSP case. FtMt-immunoreactivity is increased and FtMt-immunoreactive aggregates and small dots are seen (arrow). E: TH-immunoreactivity in the SNc of a PSP case. F: Merged images of FtMt- and TH-immunoreactivity in the SNc of a PSP case. FtMt-immunoreactive structures are observed in TH-positive neurons (arrow). G: The density of TH-positive neurons in the SNc and ventral tegmental area (VTA) in control and PSP cases ( $p=$ 0.07). H: The density of FtMt-positive neurons in the SNc and VTA in control and PSP cases $(p=0.29)$. I: Percentage of double positive neurons for FtMt and TH to total TH-immunoreactive neurons in the SNc and VTA $(p=0.343)$. J: Percentage of FtMt-positive and TH-negative neurons to total FtMt-immunoreactive neurons in the SNc and VTA in control and PSP ceases $\left(p=0.018\right.$ ). Data are presented as the mean \pm SEM. ${ }^{*} p<0.05$ (unpaired t-test). Bars $=50 \mu \mathrm{m}$.

immunoreactive structures were also observed in both the SNc (Fig. 5E) and VTA (Fig. 5H). The number of pTauimmunoreactive structures was much higher than that of FtMt-immunoreactive structures (Fig. 5D, E, G, H). In the SNc and VTA, pTau-immunoreactive structures consisted of large globose-type tangles (arrows in Fig. 5E and H) and many fine threads or small dots (arrowheads in Fig. $5 \mathrm{E}$ and $\mathrm{H}$ ). FtMt-immunoreactive dots were colocalized with some of the pTau-immunoreactive structures (arrows and arrowheads in Fig. 5). At high magnification, small FtMt-immunoreactive dots were colocalized in large pTau tangles in the SNc (arrows in Fig. 5D', E', F') and VTA (arrows in Fig. 5G', H', I'). In the SNc and VTA, 45.3\% of pTau-immunoreactive globose-type tangles contained FtMt-immunoreactive dots.

\section{Discussion}

We investigated the immunohistochemical localization of FtMt in the midbrain of patients with PSP and control cases using the mouse monoclonal antibody C65-2 directed against human FtMt. In control cases, FtMt immunoreactivity was localized to the cell bodies of some neuromelaninpositive neurons in the SNc and VTA. Our results are consistent with other studies $[27,28]$. The results from the in situ hybridization revealed that FtMt is primarily expressed in neurons [22]. Double-immunofluorescence histochemistry confirmed that $85.5 \%$ of TH-positive neurons were colocalized with FtMt. The results are consistent with our previous study, which showed that $88.2 \%$ of total TH-positive neurons contained FtMt in human SNc [28]. 

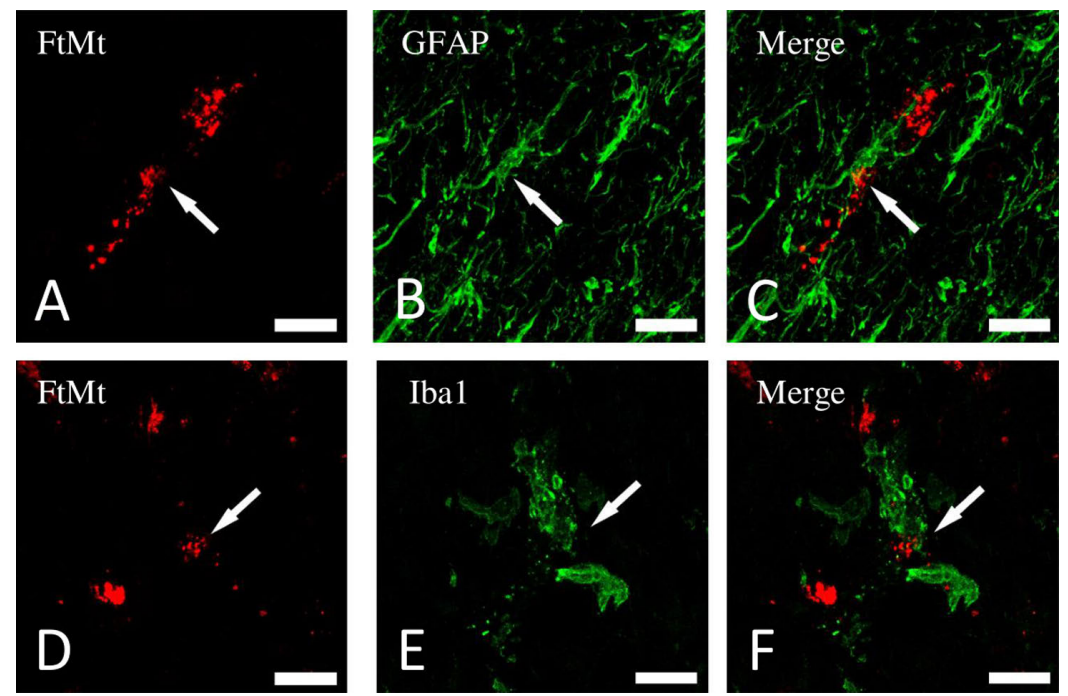

Fig. 4. Double immunofluorescent histochemistry of mitochondrial ferritin (FtMt) and glial fibrillary acidic protein (GFAP) (A-C) or Iba1 (D-F) in the substantia nigra pars compacta (SNc) of a PSP case (PSP-2). A: FtMt-immunoreactivity in the SNc. B: GFAP-immunoreactivity in the SNc. C: Merged images of FtMt- and GFAP-immunoreactivity in the SNc. Colocalization of both FtMt- and GFAP-immunoreactivity (arrow). D: FtMt-immunoreactivity in the SNc. E: Iba1-immunoreactivity in the SNc. F: Merged images of FtMt- and Iba1-immunoreactivity in the SNc. IbaI-immunoreactive cells appear to surround FtMt-positive dots. A few Iba-immunoreactive cells contain FtMt-positive dots $($ arrow). Bars $=50 \mu \mathrm{m}$.

There was no difference in the distribution patterns of FtMt in the midbrain between control cases, but the intensity of FtMt immunoreactivity differed among cases. Patients with a long postmortem time showed weak staining (Table 1).

In patients with PSP, FtMt immunoreactivity dramatically increased in neuronal cell bodies. In addition, some positive dots were observed outside the neurons. These dots often co-localized with GFAP or Iba1. The reason for which FtMt has been detected in astrocytes and microglia remains unknown. Astrocytes and microglia may phagocytose FtMt released from dead neurons. Further studies are required to shed light on this issue. It is well known that PSP cases show loss of dopaminergic neurons in the substantia nigra that project to the putamen [8]. Although the number of TH-positive neurons tended to be reduced in PSP cases in this study, there was no significant difference between PSP and control cases $(p=0.07)$. The discrepancy may be due to the small number of cases and the large variability among cases.

The ratio of double-positive neurons for FtMt and $\mathrm{TH}$ to total TH-positive neurons was not significantly different between the control and PSP cases $(p=0.343)$. Interestingly, the ratio of FtMt-positive and TH-negative neurons to total FtMt-positive neurons was significantly increased in PSP cases $(p=0.018)$. the reason remains unclear in this study. However, several possibilities are raised. One possibility is that TH-positive neurons may be more vulnerable than TH-negative neurons in PSP patients, and FtMtpositive neurons without TH remain. Another possibility is that FtMt may be induced in TH-negative neurons by proinflammatory cytokines in PSP cases. In order to clarify this issue, further studies are needed.

Previous studies have reported that FtMt expression is induced by ROS and inflammatory cytokines, such as TNF- $\alpha$, IL-1 $\beta$, and IL- 6 , in the human neuroblastoma cell line IMR-32 [22, 26]. Moreover, ROS and inflammatory cytokines are involved in the pathophysiology of PSP [2]. Our findings showing increased FtMt immunoreactivity in the midbrains of patients with PSP are consistent with these results. FtMt was found to inhibit TNF- $\alpha$-induced apoptosis [26] and erastin-induced ferroptosis [23], suggesting that FtMt inhibits inflammatory and oxidative stress-induced neuronal cell damage.

The involvement of tau aggregation, including globose-type tangles and tuft shaped astrocytes, is a hallmark of PSP $[4,10,13,21,29]$. Consistent with the results of other studies $[5,10]$, our results demonstrated a massive deposition of pTau in the midbrains of patients with PSP. In the SNc and VTA, FtMt immunoreactivity is often colocalized with pTau. These results suggest that FtMt is involved in tau pathology in patients with PSP. As reported in a previous paper [10], many pTau-immunoreactive structures were observed in the superior colliculus of PSP cases. However, FtMt immunoreactivity was not detected in superior colliculus. These results suggest that FtMt is not essential for tau aggregation in PSP.

Our findings revealed that FtMt expression was increased in dopaminergic neurons in the SNc and VTA. These results suggest that FtMt is involved in pathological processes in dopaminergic neurons in PSP. Iron is required for dopamine synthesis, and dopamine produces ROS during metabolism. FtMt plays an important role in regulating iron levels in neurons [12, 25]. A previous study reported that FtMt showed neuroprotective effects on 6hydroxydopamine-induced dopaminergic cell damage [19]. Therefore, revealing the role of FtMt in regulating iron 


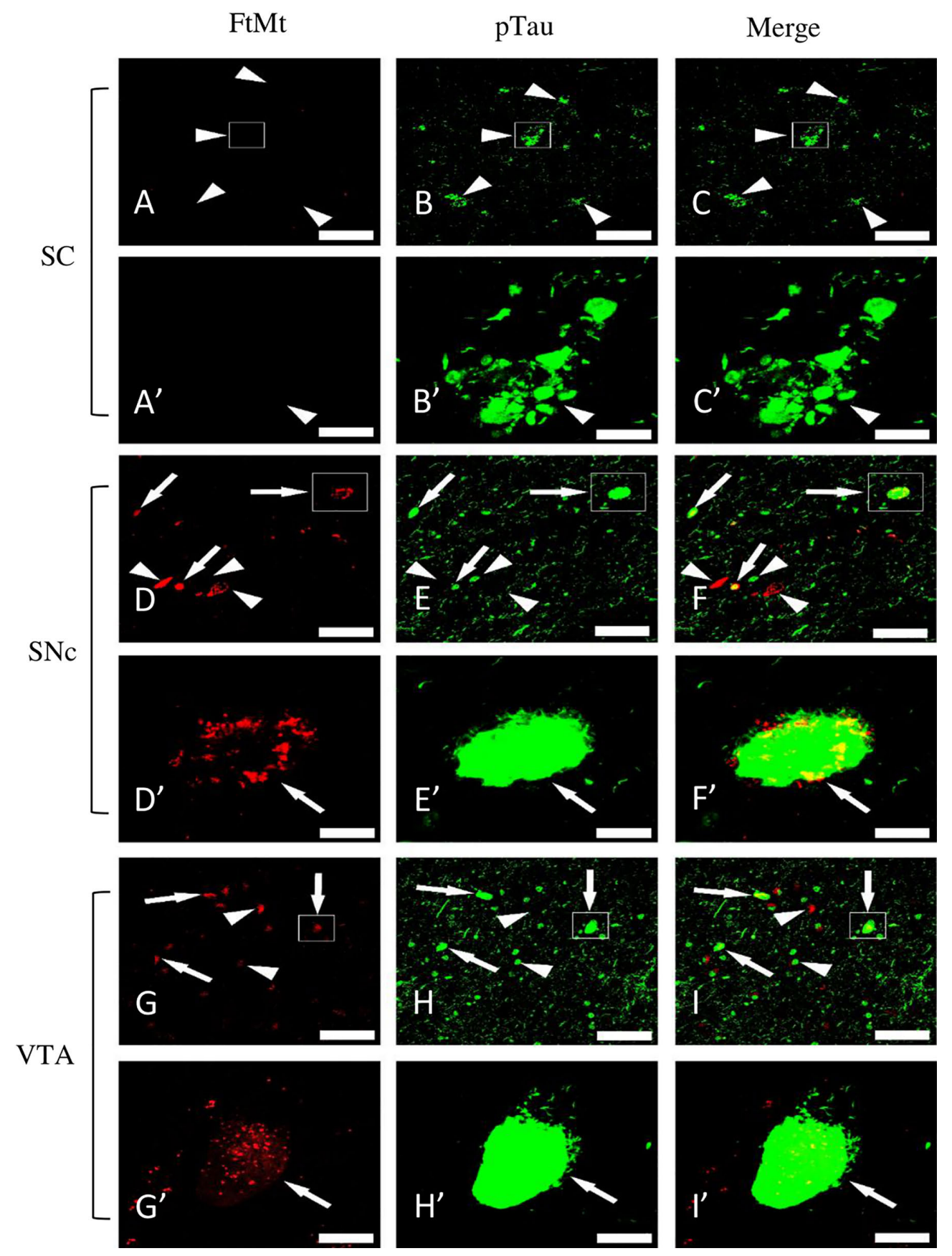

Fig. 5. pTau-immunoreactivity and mitochondrial ferritin (FtMt)-immunoreactivity in the superior colliculus (SC) (A-C), substantia nigra pars compacta (SNc) (D-F') and ventral tegmental area (VTA) (G-I') of a PSP case (PSP-2). A: No FtMt-immunoreactivity is seen in the SC. A': High-magnification image of the boxed area in A. B: pTau-immunoreactivity is prominently observed in the SC (arrowheads). B': High-magnification image of the boxed area in B. C: There was no colocalization of pTau and FtMt immunoreactivity in the SC (arrowheads). C': High-magnification image of the boxed area in C. D: FtMt-positive dots in the SNc. D': High-magnification image of the boxed area in D. E: Globose-type tangles positive for pTau in the SNc. In addition, pTau-immunoreactive threads and dots can also be seen. E': High-magnification image of the boxed area in D. F: Merged image of FtMtimmunoreactivity and pTau-immunoreactivity in the SNc. Some pTau-immunoreactive structures contain FtMt immunoreactivity in the SNc (arrows). FtMt-immunoreactive structures without pTau (arrowheads). F': High-magnification image of the boxed area in F. G: FtMt-immunoreactivity in the VTA. Prominent FtMt-immunoreactivity can be observed (arrows). G': High-magnification image of the boxed area in G. H: pTau-immunoreactivity in the VTA (arrows). H': High-magnification image of the boxed area in H. I: Merged image of FtMt- and pTau-immunoreactivity in the VTA. Some pTau-immunoreactive structures contain FtMt-immunoreactivity in the VTA (arrows). FtMt-immunoreactive structures without pTau-immunoreactivity (arrowheads). I': High-magnification image of the boxed area I. Some FtMt-immunoreactive dots can be seen in a pTau-positive globose-type tangle (arrow). Bars $=50 \mu \mathrm{m}$. 
levels in dopaminergic neurons in PSP is of great interest.

In summary, we performed an immunohistochemical analysis of FtMt in the midbrain of patients with PSP and controls using the human FtMt antibody C65-2. An increase in FtMt immunoreactivity was observed in dopaminergic neurons, as well as in some astrocytes and microglia in the SNc of patients with PSP. In the SNc and VTA, FtMt immunoreactivity was often co-localized with pTau. These results suggest that FtMt is involved in the pathological processes of PSP.

\section{Conflicts of Interest}

The authors declare that there are no conflicts of interest.

\section{Acknowledgments}

This study was supported by JSPS KAKENHI Grant Numbers JP17H03560 (I.T.) and JP19K20670 (T.K.).

\section{References}

1. Abdelalim, E. M. and Tooyama, I. (2011) Mapping of NPR-B immunoreactivity in the brainstem of Macaca fascicularis. Brain Struct. Funct. 216; 387-402.

2. Alster, P., Madetko, N., Koziorowski, D. and Friedman, A. (2020) Microglial activation and inflammation as a factor in the pathogenesis of Progressive Supranuclear Palsy (PSP). Front. Neurosci. 14; 893.

3. Aoyama, K., Matsubara, K. and Kobayashi, S. J. (2006) Aging and oxidative stress in progressive supranuclear palsy. Eur. J. Neurol. 13; 89-92.

4. Armstrong, M. J. (2018) Progressive supranuclear palsy: An Update. Curr. Neurol. Neurosci. Rep. 18; 12.

5. Armstrong, R. A. (2011) Visual signs and symptoms of progressive supranuclear palsy. Clin. Exp. Optom. 94; 150-160.

6. Boxer, A. L., Yu, J. T., Golbe, L. I., Litvan, I., Lang, A. E. and Höglinger, G. U. (2017) New diagnostics and therapeutics for progressive supranuclear palsy. Lancet Neurol. 16: 552-563.

7. Bresgen, N. and Eckl, P. M. (2015) Oxidative stress and the homeodynamics of iron metabolism. Biomolecules 5; 808-847.

8. Dickson, D. W. (2012) Parkinson's disease and parkinsonism: Neuropathology. Cold Spring Harb. Perspect. Med. 2; a009258.

9. Drysdale, J., Arosio, P., Invernizzi, R., Cazzola, M., Volz, A., Corsi, B., et al. (2002) Mitochondrial ferritin: a new player in iron metabolism. Blood Cells Mol. Dis. 29; 376-383.

10. Dugger, B. N., Tu, M., Murray, M. E. and Dickson, D. W. (2011) Disease specificity and pathologic progression of tau pathology in brainstem nuclei of Alzheimer's disease and progressive supranuclear palsy. Neurosci. Lett. 491; 122-126.

11. Ebashi, M., Ito, Y., Uematsu, M., Nakamura, A., Hirokawa, K., Kamei, S., et al. (2019) How to demix Alzheimer-type and PSPtype tau lesions out of their mixture-hybrid approach to dissect comorbidity. Acta Neuropathol. Commun. 7; 71.

12. Gao, G. and Chang, Y.-Z. (2014) Mitochondrial ferritin in the regulation of brain iron homeostasis and neurodegenerative diseases. Front. Pharmacol. 5; 19.

13. Gerson, J. E., Sengupta, U., Lasagna-Reeves, C. A., GuerreroMuñoz, M. J., Troncoso, J. and Kayed, R. (2014) Characterization of tau oligomeric seeds in progressive supranuclear palsy. Acta Neuropathol. Commun. 2; 1-9.

14. Kageyama, Y., Saito, A., Pletnikova, O., Rudow, G. L., Irie, Y., An, Y., et al. (2018) Amyloid $\beta$ toxic conformer has dynamic localization in the human inferior parietal cortex in absence of amyloid plaques. Sci. Rep. 8; 16895.

15. Kawashima, M., Miyake, M., Kusumi, M., Adachi, Y. and Nakashima, K. (2004) Prevalence of progressive supranuclear palsy in Yonago, Japan. Mov. Disord. 19; 1239-1240.

16. Levi, S. and Arosio, P. (2004) Mitochondrial ferritin. Int. J. Biochem. Cell Biol. 36; 1887-1889.

17. Santambrogio, P., Biasiotto, G., Sanvito, F., Olivieri, S., Arosio, P. and Levi, S. (2007) Mitochondrial ferritin expression in adult mouse tissues. J. Histochem. Cytochem. 55; 1129-1137.

18. Schrag, A., Ben-Shlomo, Y. and Quinn, N. (1999) Prevalence of progressive supranuclear palsy and multiple system atrophy: A cross-sectional study. Lancet $354 ; 1771-1775$.

19. Shi, Z.-H., Nie, G., Duan, X.-L., Rouault, T., Wu, W.-S., Ning, B., et al. (2010) Neuroprotective mechanism of mitochondrial ferritin on 6-hydroxydopamine-induced dopaminergic cell damage: Implication for neuroprotection in Parkinson's disease. Antioxid. Redox Signal. 13; 783-796.

20. Spotorno, N., Acosta-Cabronero, J., Stomrud, E., Lampinen, B., Strandberg, O. T., van Westen, D., et al. (2020) Relationship between cortical iron and tau aggregation in Alzheimer's disease. Brain 143; 1341-1349.

21. Togo, T. and Dickson, D. W. (2002) Tau accumulation in astrocytes in progressive supranuclear palsy is a degenerative rather than a reactive process. Acta Neuropathol. 104; 398-402.

22. Wang, L., Yang, H., Zhao, S., Sato, H., Konishi, Y., Beach, T. G., et al. (2011) Expression and localization of mitochondrial ferritin mRNA in Alzheimer's disease cerebral cortex. PLoS One 6; e225.

23. Wang, Y.-Q., Chang, S.-Y., Wu, Q., Gou, Y.-J., Jia, L., Cui, Y.-M., et al. (2016) The protective role of mitochondrial ferritin on erastin-induced ferroptosis. Front. Aging Neurosci. 8; 308.

24. Yamamoto, A., Shin, R. W., Hasegawa, K., Naiki, H., Sato, H., Yoshimasu, F., et al. (2002) Iron (III) induces aggregation of hyperphosphorylated $\tau$ and its reduction to iron (II) reverses the aggregation: implications in the formation of neurofibrillary tangles of Alzheimer's disease. J. Neurochem. 82; 1137-1147.

25. Yang, H., Yang, M., Guan, H., Liu, Z., Zhao, S., Takeuchi, S., et al. (2013) Mitochondrial ferritin in neurodegenerative diseases. Neurosci. Res. 77; 1-7.

26. Yang, H., Guan, H., Yang, M., Liu, Z., Takeuchi, S., Yanagisawa, D., et al. (2015) Upregulation of mitochondrial ferritin by proinflammatory cytokines: implications for a role in Alzheimer's disease. J. Alzheimers Dis. 45; 797-811.

27. Yang, M., Yang, H., Guan, H., Bellier, J.-P., Zhao, S. and Tooyama, I. (2016) Mapping of mitochondrial ferritin in the brainstem of Macaca fascicularis. Neuroscience 328; 92-106.

28. Yang, M., Yang, H., Guan, H., Kato, T., Mukaisho, K., Sugihara, H., et al. (2017) Characterization of a novel monoclonal antibody against human mitochondrial ferritin and its immunohistochemical application in human and monkey substantia nigra. Acta Histochem. Cytochem. 50; 49-55.

29. Yoshida, M. (2014) Astrocytic inclusions in progressive supranuclear palsy and corticobasal degeneration. Neuropathology 34; 555-570.

This is an open access article distributed under the Creative Commons License (CC-BY-NC), which permits use, distribution and reproduction of the articles in any medium provided that the original work is properly cited and is not used for commercial purposes. 